\title{
Dielectric Resonator Method for Measuring the Electrical Conductivity of Carbon Nanotubes from Microwave to Millimeter Frequencies
}

\author{
James Baker-Jarvis, ${ }^{1}$ Michael D. Janezic, ${ }^{1}$ and John H. Lehman ${ }^{2}$ \\ ${ }^{1}$ Electromagnetics Division, National Institute of Standards and Technology (NIST), Boulder, CO 80305-3328, USA \\ ${ }^{2}$ Optoelectronics Division, National Institute of Standards and Technology (NIST), Boulder, CO 80305-3328, USA
}

Received 27 February 2007; Accepted 21 June 2007

Recommended by Christian Brosseau

We describe a dielectric resonator-based measurement method for determining the electrical conductivity of carbon nanotubes at microwave to millimeter frequencies. This measurement method is not limited by the metal conductor contact resistances or impedance mismatch commonly encountered in the measurement of single nanotubes. The measurement of carbon nanotubes yielded conductivities of approximately $0.08 \times 10^{7} \mathrm{~S} / \mathrm{m}$.

Copyright (c) 2007 James Baker-Jarvis et al. This is an open access article distributed under the Creative Commons Attribution License, which permits unrestricted use, distribution, and reproduction in any medium, provided the original work is properly cited.

\section{INTRODUCTION}

The objective of this study is to develop an accurate method to measure the electrical conductivity of carbon nanotubes in the gigahertz frequency range that minimizes the effects of contact resistance and impedance mismatch. The application of the high-frequency technique we develop in this paper is new and does not require connections across metallic transmission-line conductors. It therefore bypasses a number of calibration issues that are encountered by the use of other methods.

The dielectric resonator method for loss determination has been used extensively for measurements of the surface resistance of superconducting thin films and for the measurement of metal resistivity [1-3]. The measurement method uses the $\mathrm{TE}_{011}$ mode of a sapphire dielectric resonator, as diagramed in Figure 1 and the resonating fixture as shown in Figure 2. This method is similar to that used by Courtney for measurements of the permittivity of rod specimens; however in this research, the permittivity is determined by measurement, and the conductor losses of the nanotubes are treated as unknown [4].

In applications where nanotubes or nanowires could be used in electrical devices, the operational frequencies would be in the microwave through millimeter bands. Therefore, the measurement of the electrical properties of nanotubes is important. The theoretical model of the electrical conductivity of nanotubes is usually based on the work of Lüttinger [5]. Over the years, the conductivity of carbon nanotubes has been studied both experimentally and theoretically by a number of different methods [6]. Some researchers have attempted to measure carbon nanotubes individually by welding the nanotubes across microconductors that have been deposited onto transmission lines. Circuit models are then used to estimate the electrical conductivity $[5,7]$. The main problem with these approaches is that there is a large impedance mismatch between the nanotube and the transmission line as well as an unknown contact resistance at the position of the weld. The method developed in this paper is not limited by these measurement artifacts. Our method yields a conductivity that is averaged over many nanotubes.

In our method, carbon nanotubes were deposited as a coating on the end-faces of the sapphire rods, and then, the resonators were fitted into a parallel-plate resonator system that allows a measurement of the quality factor $(Q)$. Changes in the $Q$ of the coated resonators over those of the bare resonators allow for the calculation of the surface resistance and electrical conductivity of bulk nanotube layers $[1,2]$.

The single-wall carbon nanotube (SWNT) samples for the present work were obtained from Southwest NanoTechnologies produced by the proprietary CoMoCAT process (S-P95-Dry). The manufacturer indicates that the material 


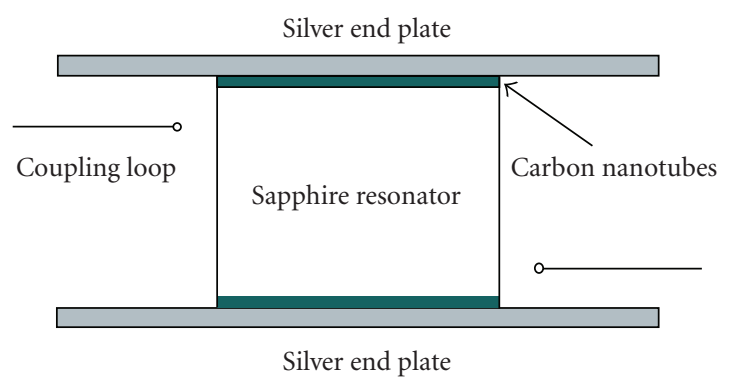

Figure 1: A parallel-plate and resonator with a layer of carbon nanotubes deposited on both ends.

content should consist of no more than $5 \%$ nonnanotube materials. The resonator coating was formed from the bulk SWNTs dispersed in chloroform, sonicated for 10 minutes and applied with an airbrush at $6.9 \mathrm{kPa}$ [8]. The proportion of carbon nanotubes to chloroform, by mass, was approximately 1 : 100. Resonant Raman Spectroscopy (RRS) of a representative sample of the SWNT coating was performed in the backscattering configuration using $7 \mathrm{~mW}$ of an argon ion laser providing $488 \mathrm{~nm}(2.54 \mathrm{eV})$ excitation. A $55 \mathrm{~mm}$ telephoto lens was employed to both focus the beam to approximately $0.25 \mathrm{~mm}^{2}$ area and to collect the Raman scattered light. The scattered light was analyzed with a $0.27 \mathrm{~m}$ grating spectrometer equipped with a liquidnitrogen-cooled, charge-coupled detector and a holographic notch filter. From this we obtained a measure of purity as quantified by the ratio of intensities of " $D$ " and " $G$ " bands, where $\mathrm{D} / \mathrm{G}=0.0342$.

In our procedure, the permittivity of the rod is calculated from the resonant frequency and used to calculate the conductivity of the coated ends of the resonator. Either one or both of the ends can be coated with the unknown nanotube material. Our approach assumes that the researcher has a sufficient amount of carbon nanotubes to coat the upper or lower surfaces of a resonator, or both, to a thickness of a few electrical skin depths. This thickness is sufficient to ensure that very little field penetrates to the silver end plates. When both faces of the sapphire cylinder are terminated with conducting end plates, the surface resistance and electrical conductivity can be calculated from measurements of the shift in $Q$ relative to those of the uncoated resonator $[1,2]$. The method is robust since specimens can be measured at various frequencies, from microwave to millimeter frequencies, by use of different size sapphire resonators. Another advantage of this method over other transmission-line methods is that fixture-metal losses are minimized.

In Section 2, we overview the theoretical model for conductivity determination. In Section 3, we introduce the measurement procedure; and in Section 4, we present measurement results.

\section{SURFACE RESISTIVITY MODEL}

This section is a short overview of the model we used to deconvolve the conductivity from measurements of the $Q$

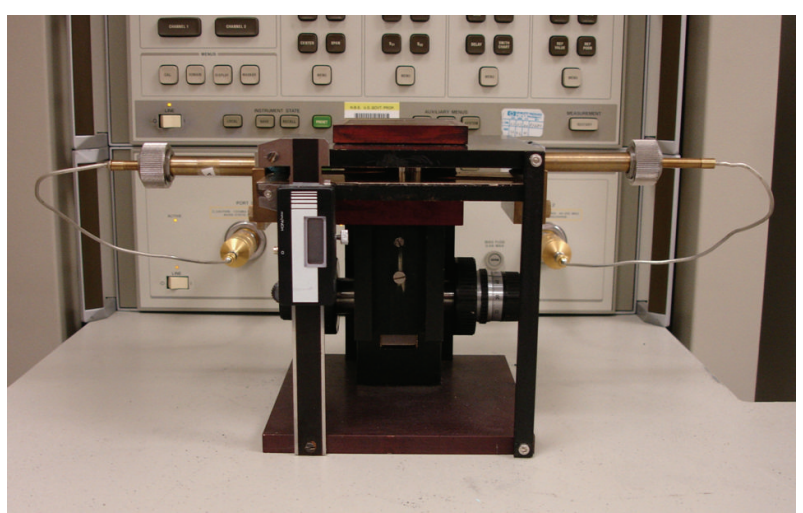

Figure 2: A parallel-plate resonator measurement fixture.

and resonant frequency. The calculation of the conductivity is based on an electromagnetic field model that uses the $Q$ and resonant frequency $f_{r}$ of the unloaded and loaded dielectric resonator to obtain an effective conductivity. A transcendental equation for the resonant frequencies is obtained by matching the tangential components of the electric and magnetic fields across the resonator-nanotube interfaces [2]:

$$
\frac{J_{1}\left(\nu_{1} a\right) K_{0}\left(\nu_{2} a\right)}{\nu_{1} a}+\frac{K_{1}\left(\nu_{2} a\right) J_{0}\left(\nu_{1} a\right)}{\nu_{2} a}=0,
$$

where $a$ denotes the radius of the sapphire rod, $\nu_{1}=$ $\sqrt{k^{2} \epsilon_{r s}-(\pi / L)^{2}}$, and $\nu_{2}=\sqrt{(\pi / L)^{2}-k^{2}}, L$ is the height of the sapphire rod, $k=\omega / c=\omega \sqrt{\epsilon_{0} \mu_{0}}, \epsilon_{r s}$ is the permittivity of the resonator, and $J, K$ denote Bessel and modified Bessel functions, respectively. The roots of (1) determine the permittivity of the rod given the measured resonant frequencies $f_{r}$. The measured $Q$ is decomposed into contributions from the nanotubes $Q_{c}$ and the losses in the dielectric $Q_{d}$ :

$$
\frac{1}{Q}=\frac{1}{Q_{c}}+\frac{1}{Q_{d}}
$$

where

$$
Q_{d}=\frac{1+R}{\tan \delta}
$$

where $\tan \delta=\epsilon^{\prime \prime} / \epsilon^{\prime}$ and

$$
R=\frac{1}{\epsilon_{r s}}\left[\frac{\nu_{1} J_{0}\left(\nu_{1} a\right)}{v_{2} K_{0}\left(v_{2} a\right)}\right]^{2} \frac{\int_{a}^{\infty} \rho K_{1}^{2}\left(\nu_{2} \rho\right) d \rho}{\int_{0}^{a} \rho J_{1}^{2}\left(\nu_{1} \rho\right)} .
$$

The surface resistance can then be calculated by use of $1 / Q_{c}=$ $1 / Q-1 / Q_{d}$ with (3) and (4) from

$$
R_{s}=240 \pi^{2} \epsilon_{r}\left[\frac{1}{Q}-\frac{\tan (\delta)}{1+R}\right] \frac{1+R}{1+\epsilon_{r s} R}\left(\frac{L}{\lambda}\right)^{3} .
$$

Once we have obtained $R_{s}$, using the knowledge that the material is nonmagnetic, we can calculate the conductivity from

$$
\sigma=\frac{\pi f_{r} \mu_{0}}{R_{s}^{2}}
$$




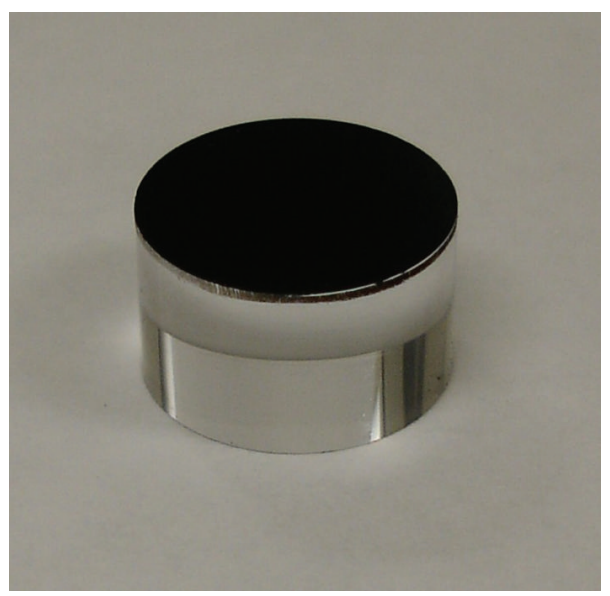

Figure 3: A dielectric resonator with carbon nanotubes deposited on the end surfaces.

The skin depth $\delta_{s}$ is the distance traveled by the fields before they are damped to $1 / e$ of their initial value. Estimation of the skin depth is important in order to predict how thick the carbon nanotube layers need to be in order to minimize field penetration. The skin depth is given by

$$
\delta_{s}=\sqrt{\frac{1}{\pi f_{r} \mu_{0} \sigma}} .
$$

\section{MEASUREMENTS}

In order to investigate the frequency dependence of the conductivity, we used two cylindrical single-crystal sapphire resonators machined to have dimensional aspect ratios yielding optimal field structures at 5 and $10 \mathrm{GHz}$.

We deposited a layer of carbon nanotubes, approximately 30 to $60 \mu \mathrm{m}$ thick on both of the ends of the sapphire resonators, as shown in Figure 3. The sapphire resonator was placed between two silver end plates in the fixture shown in Figure 2. The coupling loops excited a $\mathrm{TE}_{011}$ resonant mode. We measured the $Q$ and resonant frequency of the $\mathrm{TE}_{011}$ mode with a network analyzer for both the coated and uncoated resonators. The losses in the carbon nanotubes produces a decrease in the $Q$ factor. We used the $Q$ and $f_{r}$ to calculate the surface resistance from (5) and thereby the effective conductivity of the carbon nanotubes from (6).

The permittivity of the sapphire resonators were $\epsilon_{r}^{\prime}=$ 9.458 and $\tan \delta=1 \times 10^{-6}$. One resonator operated at $4.98 \mathrm{GHz}$ and had a diameter of $23.178 \mathrm{~mm}$ and a height of $15.452 \mathrm{~mm}$. The other resonator operated at $9.95 \mathrm{GHz}$ and had a diameter of $11.589 \mathrm{~mm}$ and a height of $7.726 \mathrm{~mm}$. Millimeter frequency measurements require smaller resonators. In order for the measurements to be consistent with the model, the layer of carbon nanotubes must be a few skin depths in thickness. Using the measured thickness of $30 \mu \mathrm{m}$, we estimate the skin depth from our measured conductivity to be less than $10 \mu \mathrm{m}$. Therefore, this thickness is adequate for the fields to decay before it reaches the cavity end walls. For comparison purposes, the skin depth of fields incident
TABLE 1: The measured effective conductivity and associated uncertainties for two sets of depositions of carbon nanotubes.

\begin{tabular}{l|ccc}
\hline Configuration & $\begin{array}{l}\text { Frequency } \\
(\mathrm{GHz})\end{array}$ & $\begin{array}{l}\text { Conductivity } \\
(\mathrm{S} / \mathrm{m})\end{array}$ & Uncertainty \\
\hline Resonator 1 & 9.958 & $1.8 \times 10^{6}$ & $U=2 \times 10^{5}$ \\
Resonator 2 & 4.988 & $1.8 \times 10^{6}$ & $U=2 \times 10^{5}$ \\
Resonator 1 & 9.958 & $0.8 \times 10^{6}$ & $U=1 \times 10^{5}$ \\
Resonator 2 & 4.988 & $0.4 \times 10^{6}$ & $U=1 \times 10^{5}$ \\
\hline
\end{tabular}

on our silver-plated fixture is approximately $0.64 \mu \mathrm{m}$. The results of the measured conductivity are displayed in Table 1.

The standard uncertainty is defined as $u\left(x_{i}\right)$, where $x_{i}$ are the independent sources of uncertainty. Uncertainties that can be calculated by statistical means are called Type A. The uncertainties for the resonator were developed by a statistical approach. The combined RSS standard uncertainty is calculated as

$$
u_{c}=\sqrt{\sum_{i}\left(u\left(x_{i}\right) \frac{\partial \theta}{\partial x_{i}}\right)^{2}} .
$$

In this paper, the measurand is $\sigma$ and we included the independent uncertainties due to the sapphire resonator dimensions, film thickness, $Q$ determination, and resonant frequency. The uncertainties of the $Q$ and thickness of the material dominated. The expanded uncertainty is $U(\theta)=k u_{c}(\theta)$, where $k$ is the coverage factor. We have used $k=2$. We noted a relatively large systematic uncertainty between different coatings of carbon nanotubes. The origins of this systematic uncertainty is probably due to the deposition process and possibly variations in nanotubes.

\section{CONCLUSIONS}

The resonator method described in this paper bypasses some of the difficulties encountered in single-strand methods. Our results yield conductivities that are slightly greater than that reported by other authors. For example, Ma $0.05 \times 10^{7} \mathrm{~S} / \mathrm{m}$ reported [9]. We believe this is due in part to variations between nanotubes and the fact that our measurements are minimally influenced by metal losses. We have observed some variations of conductivity from different carbon nanotube batches probably due to variations in the carbon nanotubes, or deposition process and thickness uncertainties. In transmission lines at micro wave frequencies, losses due to metal influences can be appreciable. The resonator method has a very low uncertainty if the thickness of the coating is known accurately and if the coating is thick enough for the fields to decay before the copper cavity endplates. Most of the contributions to the uncertainties in Table 1 are due to these factors. Some limitations and restrictions of our method include the amount of nanotube material required, variations in the thickness of the carbon-nanotube layer; and the method yields an ensemble-averaged conductivity. This method should work equally well for any type of nanowire. 


\section{REFERENCES}

[1] J. Krupka, M. Klinger, M. Kuhn, et al., "Surface resistance measurements of HTS films by means of sapphire dielectric resonators," IEEE Transactions on Applied Superconductivity, vol. 3, no. 3, pp. 3043-3048, 1993.

[2] Z.-Y. Shen, C. Wilker, P. Pang, W. L. Holstein, D. Face, and D. J. Kountz, "High $\mathrm{T}_{c}$ superconductor-sapphire microwave resonator with extremely high Q-values up to $90 \mathrm{~K}$," IEEE Transactions on Microwave Theory and Techniques, vol. 40, no. 12, pp. 2424-2431, 1992.

[3] J. Baker-Jarvis, M. D. Janezic, B. Riddle, et al., "Measuring the permittivity and permeability of lossy materials: solids, liquids, metals, building materials, and negative-index materials," Tech. Rep. 1536, National Institute of Standards and Technology, Boulder, Colo, USA, February 2005.

[4] W. E. Courtney, "Analysis and evaluation of a method of measuring the complex permittivity and permeability of microwave insulators," IEEE Transactions on Microwave Theory and Techniques, vol. 18, no. 8, pp. 476-485, 1970.

[5] P. J. Burke, "Lüttinger liquid theory as a model of the gigahertz electrical properties of carbon nanotubes," IEEE Transactions on Nanotechnology, vol. 1, no. 3, pp. 129-144, 2002.

[6] P. Singjai, S. Changsarn, and S. Thongtem, "Electrical resistivity of bulk multi-walled carbon nanotubes synthesized by an infusion chemical vapor deposition method," Materials Science and Engineering A, vol. 443, no. 1-2, pp. 42-46, 2007.

[7] P. Chiu and I. Shih, "A study of the size effect on the temperature-dependent resistivity of bismuth nanowires with rectangular cross-sections," Nanotechnology, vol. 15, no. 11, pp. 1489-1492, 2004.

[8] J. H. Lehman, C. Engtrakul, T. Gennett, and A. C. Dillon, "Single-wall carbon nanotube coating on a pyroelectric detector," Applied Optics, vol. 44, no. 4, pp. 483-488, 2005.

[9] R. Z. Ma, C. L. Xu, B. Q. Wei, J. Liang, D. H. Wu, and D. J. Li, "Electrical conductivity and field emission characteristics of hot-pressed sintered carbon nanotubes," Materials Research Bulletin, vol. 34, no. 5, pp. 741-747, 1999. 

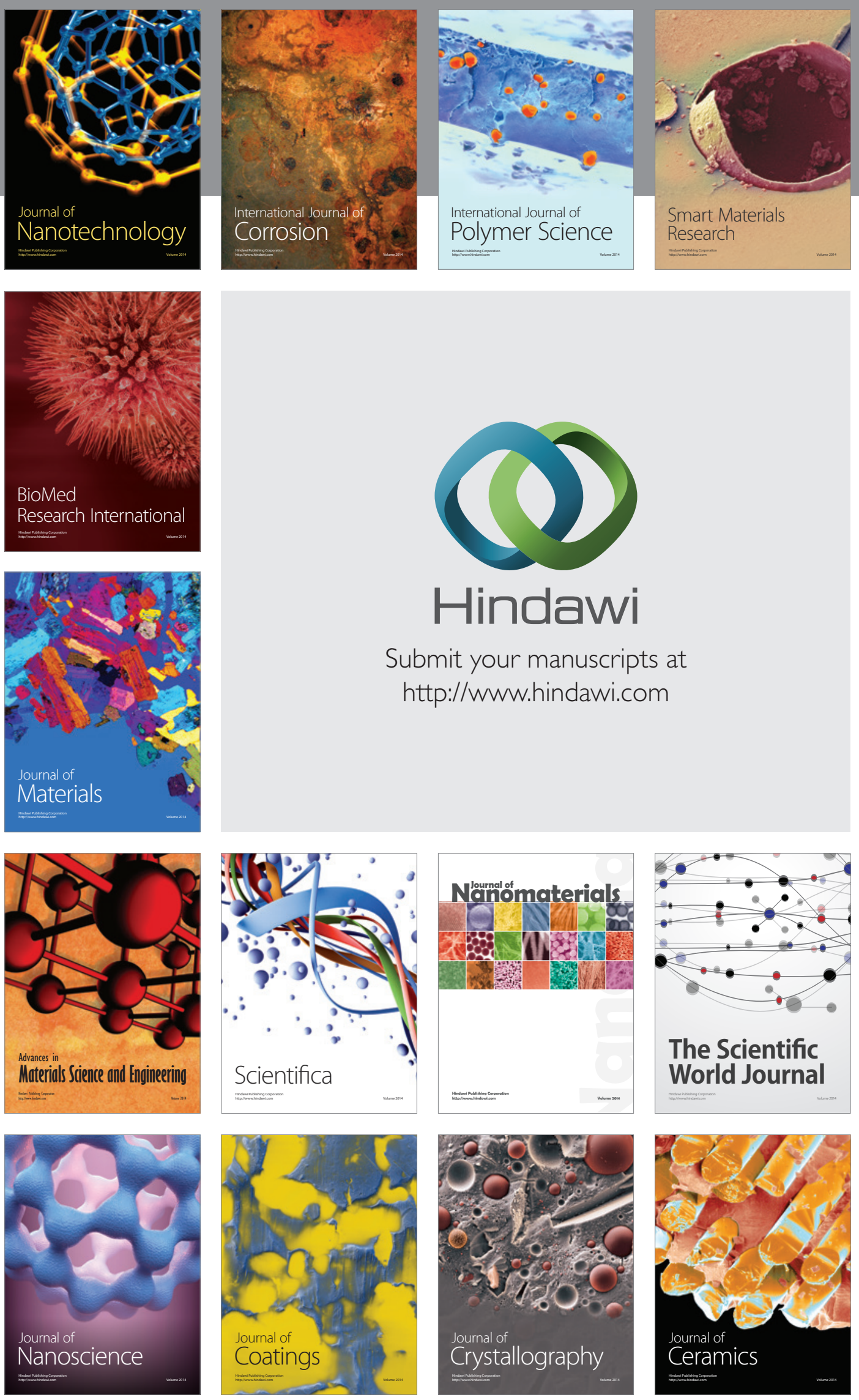

The Scientific World Journal

Submit your manuscripts at

http://www.hindawi.com

\section{World Journal}

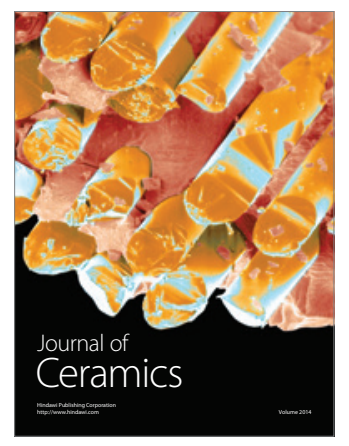

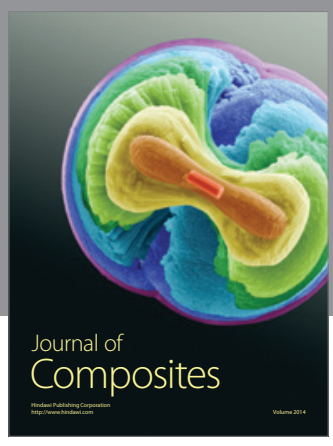
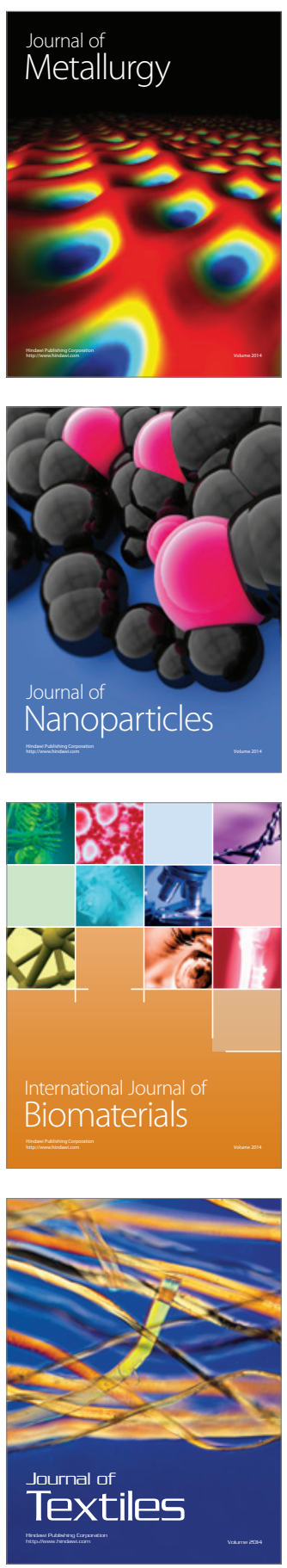